\title{
Obstructive Sleep Apnea and Diabetes Mellitus: A Bitter Combo
}

\author{
${ }^{1}$ Ketaki Utpat, ${ }^{2}$ Unnati Desai, ${ }^{3}$ Jyotsna M Joshi
}

\begin{abstract}
Obstructive sleep apnea (OSA) is a sleep-related breathing disorder (SRBD) hallmarked by upper airway collapsibility and obstruction leading to nocturnal hypoxia and sleep arousals. Diabetes mellitus (DM) is a chronic medical malady which stems from an aberration in the glucose metabolism leading to multisystemic complications and eventually ends organ damage. Both these disorders are independently major public health concerns by virtue of their momentous morbidity, mortality and healthcare albatross. The liaison between these two leviathans is enigmatic and multifarious. Prima facie they share a common predisposing factor of obesity. OSA is associated with impairment of glucose metabolism and predisposes the individual to the development of DM.
\end{abstract}

Moreover DM serves as a risk factor for OSA in conjunction with the other components of the metabolic syndrome. Untreated DM leads to aggravation of the severity of OSA while untreated OSA leads to suboptimal glycemic control and propagates complications of DM. This intricate relationship between DM and OSA is a fertile and deficiently fathomed terrain for contemplative research. We hereby make a humble endeavor to decipher and simplify the Daedalean association.

Keywords: DM, OSA, SRDB

How to cite this article: Utpat K, Desai U, Joshi JM. Obstructive Sleep Apnea (OSA) and Diabetes Mellitus: ABitter Combo. Indian Sleep Med 2018;13(3):48-52.

Source of support: Nil

Conflict of interest: None

\section{INTRODUCTION AND BACKGROUND}

OSA is a platitudinous SRBD which is characterized by recurrent nocturnal upper airway obstruction (UAO) leading to the cessation of airflow despite of continued respiratory efforts. This, in turn, leads to hypoxia and sleep fragmentation with the ensuing oxidative stress and daytime hyper somnoloscence. ${ }^{1}$ The prevalence of OSA has been estimated to be approximately in the range of from $0.3-5 \% .{ }^{1}$ OSA affects $2-4 \%$ of middle-aged men

${ }^{1}$ Assistant Professor, ${ }^{2}$ Associate Professor, ${ }^{3}$ Professor and Head

1-3Department of Pulmonary Medicine, Topiwala National Medical College, B.Y.L. Nair Hospital, Maharashtra, India

Corresponding Author: Jyotsna M Joshi, Professor and Head, Department of Pulmonary Medicine T. N. Medical College and B. Y. L. Nair Hospital, Maharashra, India, Tele: 02223027643, Email: drjoshijm@gmail.com and $1-2 \%$ of middle-aged women. However, despite this noteworthy occurrence, a majority of affected individuals remain undiagnosed due to lack of awareness and the heterogeneous presentations. Untreated OSA can potentially have horrendous metabolic, cardiovascular, neurocognitive and psychiatric complications. The addition of obesity and obesity hypoventilation syndrome (OHS) amplifies these intricacies. ${ }^{2} \mathrm{DM}$ is an old-chestnut major medical disease with its pathogenesis enrooted in a defect in the glucose metabolism with variable degrees of insulin resistance (IR) and pancreatic b-cell dysfunction. This is regulated by a complex interplay of genetic and environmental factors. ${ }^{3}$ It is associated with a high morbidity, mortality, economic burden and significant complications due to the progressive end-organ damage. The global prevalence of DM is 415 million with $46.5 \%$ of these cases lingering undiagnosed as per the International Diabetes Federation (IDF) Diabetes Atlas 7th edition, Update for $2015 .{ }^{4} \mathrm{DM}$ can be majorly divided as Type $1 \mathrm{DM}$ (T1DM) and Type $2 \mathrm{DM}$ (T2DM). T1DM results from insulin deficiency which occurs as the squeal of destruction of the insulin-producing pancreatic beta cells. It most commonly presents in childhood, but approximately $25 \%$ of cases are diagnosed in adults. T2DM is the most common form of DM. It is characterized by disorders of insulin action and insulin secretion, either singly or in combination. The risk of developing T2DM increases with age, obesity, and physical inactivity with a strong familial aggregation. Both these major maladies of OSA and DM can be independently considered as global pandemics that cast a huge toll on the precious lives and encumber the health system. Moreover when these two behemoths coexist, the association becomes fateful. The pathogenesis, clinical and metabolic manifestations, complications and management issues are intricately related in various aspects. We hereby venture to unscramble this association in a nutshell in this review article. We did an exhaustive literature search and compiled selected articles pertaining to our topic of interest. Our databases consisted of various literature pearls from Pubmed, Scopus and Google Scholar. These included meta-analyses, randomized and non randomized control trials, case-control and cohort studies and review articles. And after scrutinizing 471 articles we could narrow down on 
41 articles. Based on these we attempted to put forth in a gist the salient aspects of this coevality.

\section{Epidemiology and Prevalence of the Combined Problem}

The association between OSA and DM has been studied exhaustively in literature. Numerous clinic-based and population studies suggest that up to $40 \%$ of patients with OSA have DM. ${ }^{5}$ Although there is sufficient clarity pertaining to the prevalence, the incidence of new-onset DM in patients with diagnosed OSA is not known. Some studies have even depicted a dose-response relation between severity of OSA in terms of apnea-hypopnea index (AHI) and the prevalence of DM. ${ }^{6}$ Whereas in a landmark study the Wisconsin sleep study, the association was noted at all degrees of OSA severity and this persisted even after adjustment for body weight. ${ }^{7}$ The Busselton health study endorsed this fact by reporting a significant independent association between moderate to severe OSA and incident diabetes over a 4-year follow-up period. ${ }^{8}$ The prevalence of OSA in patients with T2DM has been variably reported to be between $40-80 \%$. As per the sleep heart health study, the prevalence of OSA in patients diagnosed to have DM was estimated to be approximately $23 \%$ while the prevalence of SDB to be $58 \% .{ }^{9}$ While in the sleep action for health in diabetes (AHEAD) study Study, the prevalence of OSA among obese diabetics was $86 \% .{ }^{10}$ The prevalence of OSA is found to be higher not only in Type 2 but also in Type 1 $\mathrm{DM}$ as per a meta-analysis which incorporated 22 studies. ${ }^{11}$ And unlike the patients with T2DM, patients with T1DM generally do not present with the garden symptoms of OSA making the diagnosis challenging. ${ }^{12}$ Thus the combined existence is voluminous in terms of prevalence as well as material in terms of consequences. ${ }^{13}$

\section{Obstructive Sleep Apnea-Definitions and Pathophysiology, Epidemiology, Clinical Features and Diagnosis}

OSA is a SRBD which emanates from recurrent airway collapsibility during sleep leading to a cessation (apnea) or a significant decrease in the airflow (hypopnea). Apnoea is defined as cessation of airflow for 10 seconds or more while hypopnoea is defined as a decrease in the amplitude of airflow of $>30 \%$ from baseline during sleep; associated with either an oxygen desaturation of greater than $3 \%$ or an arousal and the event last 10 seconds or longer. OSA is the occurrence of an average five or more episodes of obstructive respiratory events per hour of sleep with either sleep-related symptoms or comorbidities or 15 such episodes without any sleep-related symptoms or co-morbidities. Obstructive sleep apnea syndrome (OSAS) is defined as OSA associated with daytime symptoms, most often excessive daytime sleepiness (EDS). ${ }^{4}$ The Indian obstructive sleep apnoea (INOSA) guidelines which are a consensus and evidence-based Indian guidelines provide an insight into the Indian Scenario, approach to OSA and the therapy implications. ${ }^{14}$ Age, sex, ethnicity, and gender appear to be the prime demographic predisposing factors. OSA prevalence in the elderly is significantly higher than that in the younger age group. Increased upper airway collapsibility with age could be the likely explanation. ${ }^{15}$ Men have two to three times increased the risk for OSA compared with women. This can be attributed to differences in sex hormones and their influence on upper airway size and ventilatory control. ${ }^{15}$ Several risk factors have been identified in the development of OSAS including craniofacial abnormalities, upper airway anatomy, smoking, alcohol, and genetic predisposition. But undoubtedly, the strongest risk factor is obesity reflected by several markers including body mass index (BMI), neck circumference, and waist-to-hip ratio. ${ }^{16}$ This could be rationalized based on several mechanisms. The increased parapharyngeal fat deposition in obesity can result in a smaller upper airway. Alteration in the neural compensatory mechanisms and blunting of chemosensitivity to oxygen $\left(\mathrm{O}_{2}\right)$ and carbon dioxide $\left(\mathrm{CO}_{2}\right)$, with resultant ventilator instability could be the other mechanisms. Clinical symptoms suggestive of OSA can be divided into nocturnal symptoms and daytime symptoms. The nocturnal symptoms include loud snoring frequently crescendo-decrescendo, witnessed breathing pauses by a bed-partner, choking or gasping during sleep, nocturia, insomnia, and paroxysmal nocturnal dyspnea. The daytime symptoms include morning headaches, (EDS), excessive irritability, memory lapses and decreased concentration. ${ }^{14}$ Confirmation of OSA warrants an overnight polysomnography (PSG), ${ }^{14}$ a time-consuming and expensive test of limited availability. PSG can be subclassified into four types depending on the number of neurocardio and respiratory channels employed and supervision by a sleep technician. The increasing recognition of OSA as a prevalent and highmortality disease has increased PSG demand. Even in developed countries, there is a long waiting period for sleep studies. Hence, to strike a golden balance between demand and availability, triaging patients as per the estimated severity and the need for urgency of sleep study becomes vital. Various pretest probability scores have been developed to screen patients for a PSG, and assessing risk for OSA like sleep apnea clinical score (SACS), the Berlin questionnaire (BQ) the Epworth sleepiness scale (ESS) which is used to assess daytime sleepiness and the STOP-Bang score which is routinely used by the anaesthetists to assess probability of OSAS in patients to be taken up for surgeries. ${ }^{17}$ Thus, the diagnosis of OSA 
involves a two-pronged approach of suspecting and risk stratification by the pretestprobability questionnaires and subsequent confirmation by a PSG.

\section{Obstructive Sleep Apnea and Diabetes Mellitus: A Complex interplay of Pathogenesis}

The pathogenesis of the coexistence is essentially bidirectional. ${ }^{18}$ Sleep disorders and sleep debt can serve as a predisposing factor for several endocrinological diseases, DM being the most salient one. The fact that OSA is an independent contributory factor for the development of glucose intolerance and insulin resistance has been documented in various studies. In a population-based cohort study, it demonstrated that individuals with OSA were more likely to have lower levels of insulin sensitivity and higher levels of fasting insulin production compared to those without OSA and the association was statistically significant. ${ }^{19}$ The fundamental rudiment which triggers the pathogenetic cascade has been postulated to be hypoxia. This further leads to a chain of events including activation of the sympathetic nervous system, ${ }^{20}$ oxidative stress, dysregulation of the hypothalamic-pituitary-adrenal (HPA) axis, elevated cortisol levels, alteration in adipokine levels, and low-grade systemic inflammation. ${ }^{21}$ This culminates in the increase in hepatic gluconeogenesis and decrease in skeletal muscle reuptake of glucose resulting in hyperglycemia. Duration and quality of sleep also pay a fecund role in the risk of development of DM. A meta-analysis demonstrated that the risk of developing T2DM was strikingly higher with all kinds of sleep durational and architectural aberrations. ${ }^{22}$ It has customarily been postulated that OSA may predispose to DM by the common etiological factor of obesity. However, the current evidence unearths that OSA may be associated with DM by mechanisms independent of the effect of obesity. ${ }^{21}$ The exact pathogenic mechanism behind DM predisposing to sleep-disordered breathing (SDB) and OSA is nebulous as compared to the me chanism by which OSA predisposes to DM. However, it has been postulated that diabetic autonomic neuropathy likely leads to ventilator dysfunction through impaired central control of breathing leading to SDB. ${ }^{23}$ This concept is endorsed by a study which has proved that the incidence of OSA in people with diabetes with autonomic neuropathy was greater than that in people with diabetes without autonomic neuropathy. ${ }^{24}$ Thus the pathogenesis seems to be protean and entangled.

\section{Obstructive Sleep Apnea and Diabetes Mellitus: The Effects of their Coexistence}

The occurrence of OSA in DM renders the glycemic control in DM arduous. Various reasons can logically explain this. A poor sleep quality due to the sleep fragmentation and the resulting EDS propagates lethargy, physical inactivity and perpetuates obesity. This in turns increases insulin resistance and worsens glycemic control. Also, an ineptly controlled DM leads to diabetic autonomic neuropathy. This can predispose to the development of OSA by ventilator instability and impaired central control of breathing. ${ }^{23}$ Thus this vicious cycle perpetuates leading to exponential deterioration of the metabolic status. Suboptimally controlled DM has been known to set the ball rolling for various microvascular complications like retinopathy, nephropathy, neuropathy and macrovascular complications such as coronary artery and cerebrovascular disease. It has also been proven that people with diabetes with OSA have a higher prevalence of these vascular complications. ${ }^{25} \mathrm{DM}$ as a causative factor for proliferative retinopathy is orthodox. Recent literature states that OSA is an independent predictor of proliferative retinopathy even after adjusting for conventional risk factors diabetic retinopathy. ${ }^{26}$ This risk is further compounded in individuals with OSA, where hypertension and obesity are comorbid conditions. This predisposition appears to be mediated by elevated levels of inflammatory markers, reduced endothelial regulatory function, and increased insulin resistance. ${ }^{27} \mathrm{~A}$ recent study by Fujita, et al. demonstrated an increase in the levels of inflammatory markerssuch as acylation stimulating protein, high sensitive $C$ reactive protein, and components of the membrane attack complex in obese T2DM patients with retinopathy. This provides an insight into the core pathogenic process. ${ }^{28} \mathrm{DM}$ is an established cause fornephropathy. The pathogenesis stems from an upregulation of protein kinase $C$ and transforming growth factor beta triggered by an increase in the angiotensin two and platelet-derived growth factor. This leads to glomerulosclerosis and progressive loss of renal function. ${ }^{29}$ Untreated OSA can lead to delayed wound healing in diabetics, and this phenomenon has been documented to improve post CPAP therapy in a study done on patients with diabetic foot. ${ }^{30}$ It has also been shown in a study that the association of T2DM and OSA casts a toll on the health-related quality of life as assessed by the STOP questionnaire with the correlation being statistically significant. ${ }^{31}$ Thus the confederated effects of these two entities are elephantine.

\section{Role of Screening}

Based on the intricate association between entities it is imperative to screen patients with OSA for DM and metabolic syndrome. A thorough history taking can do this, anthropometry, blood pressure measurement and fasting lipids and glucose followed with a glucose tolerance test (OGTT). This is simple bedside inexpensive and doable. Due to the screening of DM patients for OSA, there could be a controversy considering the ambiguity in pathogenesis 
and the variable responses in glycemic control with CPAP therapy. However, it is worthwhile eliciting a history of classical symptoms of OSA like witnessed apnoeas, heavy snoring or daytime sleepiness. In simple words endeavor to find out a "sleepy snorer" should be honestly made. This becomes extremely relevant particularly in obese subset ${ }^{32}$ and in patients with chronic kidney disease ${ }^{33}$ as the incidence of OSA is significantly higher in these cohorts. Those suspected to be having OSA can be subjected to the further pre-test probability tests and depending on the results of the same triaged for a PSG. A study has documented that 738 out of 818 diabetics were found to be high risk for OSA when screened by the pretest probability scores and $90 \%$ of those turned out to be positive for OSA on confirmation by a PSG. ${ }^{34}$ This justifies the role of opportune screening. All possible attempts should be made to increase the awareness about the need for screening this target group and even in the presence of sufficient awareness, time and resource constraints are the major hurdles to effective screening and identification which need to be duly addressed. ${ }^{35}$

\section{Therapy Implications}

Continuous positive airway pressure (CPAP) therapy is the bulwark treatment of OSA by its mechanism of action as a dynamic splint. Other modalities of therapy like oral appliances or surgery are resorted to only in a minor chunk that have anatomical defects of upper airway or do not respond to CPAP or are intolerant to CPAP therapy. ${ }^{14}$ The coexistence of OSA and DM has multifaceted therapy implications and the treatment goes hand in glove. The control of one disease is directly or indirectly related to optimisation of therapy of the other. Literature is incongruous for consensus on whether treatment of OSA with CPAP in patients who have diabetes has a beneficial effect in terms of glucose tolerance, insulin sensitivity or delay in diabetic complications. There are randomised control trials as well as meta-analysis which do not show a significant improvement in any of these parameters. ${ }^{36,37}$

On the contrary, there are studies which show a promising role of CPAP in the form of improved insulin sensitivity and improved $\mathrm{HbA} 1 \mathrm{c}$ levels. ${ }^{38}$ The efficacy of CPAP has been demonstrated to be greater in patients with severe OSA. ${ }^{39}$ Also as per a meta-analysis, the use of CPAP in pre-diabetics or individuals with impaired glucose tolerance reduces the risk of development of florid diabetes as gauged by an alteration in the homeostasis model assessment of insulin resistance (HOMAIR). ${ }^{40}$ This diversity in literature can be partly attributed to the variations in study designs and methodologies and confounders like metabolic syndrome and obesity which need to be taken into consideration. Considering this heterogeneity, the role of lifestyle modification, physical activity, and weight loss also becomes a pertinent factor in the overall control of the duet than just CPAP alone. ${ }^{41}$ The therapy thus requires a holistic and multidisciplinary approach involving a meticulous patient counseling, lifestyle modification, dietary and physiotherapy advice, pharmacotherapy with oral hypoglycemics or insulin and CPAP therapy. Pulmonary rehabilitation and psychiatric support are equally crucial. Once on therapy the patients need to be monitored timely from both the OSA as well as the glycemic aspect.

\section{CONCLUSION}

The relationship between diabetes and OSA is bidirectional and complex. It has solemn clinical, endocrinological, metabolic, economical and psychological implications.OSA, obesity, and DM appear to be the part of a vicious circle where one synergizes and compounds the effect of another. The mechanisms involved are labyrinthine. Lifestyle modification, judicious use of oral hypoglycemic agents, increasing awareness amongst treating physicians, routine employment of pretest probability scores, lowering the threshold for performing a PSG, and timely initiation of CPAP are the cornerstones of successful management.

\section{REFERENCES}

1. Franklin KA, Lindberg E., et al. Obstructive sleep apnea is a common disorder in the population-a review on the epidemiology of sleep apnea. J Thorac Dis. 2015;7:1311-1322.

2. Lacedonia D, Carpagnano GE, Patricelli G, Carone M, Gallo C, Caccavo I, et al. Prevalence of comorbidities in patients with Obstructive sleep apnoea syndrome, overlap syndrome and obesity hypoventilation syndrome. ClinRespir J 2018; 5: 1905-1911.

3. Stumvoll M, Goldstein BJ, Van Haeften TW et al. Type 2 diabetes: principles of pathogenesis and therapy. Lancet 2005;365:1333-1346.

4. Raju YS, Swaroopa D, Yadati R, Alekhya A, et al. Diabetes mellitus and obstructive sleep apnoea: implications for clinicians. J Clin Sci Res 2016;5:225-233.

5. Botros N, Concato J, Mohsenin V, Selim B, Doctor K, Yaggi $\mathrm{HK}$, et al. Obstructive sleep apnea as a risk factor for Type 2 diabetes. Am J Med 2009; 122: 1122-1127.

6. Ronksley PE, Hemelgarn BR, Heitman SJ, Hanly PJ, Faris PD, Quan H, et al. Obstructive sleep apnoea is associated with diabetes in sleepy subjects. Thorax 2009;64:834-839.

7. Reichmuth KJ, Austin D, Skatrud JB, Young T, et al. Association of sleep apnea and type II diabetes: a population-based study. Am J Respir Crit Care Med 2005;172:1590-1595.

8. Marshall NS, Wong KK, Phillips CL, Liu PY, Knuiman MW, Grunstein RR, et al. Is sleep apnea an independent risk factor for prevalent and incident diabetes in the Busselton Health Study? J Clin Sleep Med 2009;5:15-20.

9. Resnick HE, Redline S, Shahar E, Gilpin A, Newman A,Walter R, et al. Diabetes and sleep disturbances: findings 
from the Sleep Heart Health Study. Diabetes Care 2003; 26 : 702-709.

10. Foster GD, Sanders MH, Millman R, ZammitG, Borradaile KE, Newman AB, et al. Sleep AHEAD Research Group. Obstructive sleep apnea among obese patients with Type 2 diabetes. Diabetes Care 2009;32:1017-1019.

11. ReutrakulS, Thakkinstian A, Anothaistintawec T, Chontong S, Borel AL, Perfect MM, et al. Sleep characteristics in Type 1 diabetes and association with glucose control: systematic review and meta-analysis. Sleep Med 2016;23:26-45.

12. Banghoej AM, Nerild HH, Kristensen PL, Pedersen- Bjergaardu, Fleischer J, Jensen AE, et al. Obstructive sleep apnea is frequent in patients with Type 1 diabetes. J Diabetes Complications 2017; 31: 156-161.

13. Tahrani AA, et al. Obstructive sleep apnoea in Diabetics Does it matter? Diab Vas Dis 2017; 14: 454-462.

14. Sharma SK, Katoch VM, Mohan A, Kadhiravan T, Elavarasi A, Ragesh R, et al. Indian Initiative on Obstructive Sleep Apnoea Guidelines Working Group; Indian Initiative on Obstructive Sleep. Consensus and evidence-based INOSA Guidelines 2014 (first edition). Indian J Med Res 2014;140:451-468.

15. Punjabi NM, et al. The epidemiology of adult obstructive sleep apnea. Proc Am Thorac Soc2008;5:136-143.

16. Gozal D, Kheirandish-Gozal L, et al. Cardiovascular morbidity in obstructive sleep apnea: oxidative stress, inflammation, and much more. Am J respircrit Care Med. 2008; 177(4): 369-375.

17. Collop NA, Anderson WM, Boehlecke B, Claman D, Goldberg R, Gottlieb DJ, et al. Portable Monitoring Task Force of the American Academy of Sleep Medicine. Clinical guidelines for the use of unattended portable monitors in the diagnosis of obstructive sleep apnea in adult patients. J Clin Sleep Med. 2007;3(7):737-747.

18. Reutrakul S, Mokhlesi B. Obstructive sleep apnea and diabetes: A state of Art Review. Chest 2017; 152: 1070-1086.

19. Hermans MP, Ahn SA, Mahadeb YP, Rousseau MF, et al. Sleep apnoea syndrome and 10-year cardiovascular risk in females with type 2 diabetes: relationship with insulin secretion and insulin resistance. Diabetes Metab Res Rev 2013; 29: 227-234.

20. Pamidi S, Wroblewski K, Broussard J, Day A, Hanlon EC,Abraham V, Tasali E, et al. Obstructive sleep apnea in young lean men: impact on insulin sensitivity and secretion. Diabetes Care 2012; 35: 2384-2389.

21. Punjabi NM, Beamer BA, et al. Alterations in Glucose Disposal in Sleep-disordered Breathing. Am J RespirCrit Care Med 2009; 179: 235-240.

22. Cappuccio FP, D’Elia L, Strazzullo P, Miller MA. Quantity and quality of sleep and incidence of Type 2 diabetes: a systematic review and meta-analysis. Diabetes Care 2010;33:414-420

23. Mondini $S$, Guilleminault $C$, et al. Abnormal breathing patterns during sleep in diabetes. Ann Neurol 1985; 17: 391-395.

24. Ficker JH, Dertinger SH, Siegfried W, König HJ, Pentz M, Sailer D, et al. Obstructive sleep apnoea and diabetes mellitus: the role of cardiovascular autonomic neuropathy. EurRespir J 1998; 11: 14-19.

25. Vishwanathan V, Ramalingam IP, Ramkrishnan N, et al. Higher prevalence of obstructive sleep apnea among people with Type 2 Diabetes Mellitus in a Tertiary Care centre. J Assoc Physicians India 2017; 65: 38-42.

26. Rudrappa S, Warren G, Idris I, et al. Obstructive sleep apnoea is associated with the development and progression of diabetic retinopathy, independent of conventional risk factors and novel biomarkers for diabetic retinopathy. $\mathrm{Br} J$ Ophthalmol 2012; 96: 1535.

27. Kato M, Roberts-Thomson P, Phillips BG, Haynes WG, Winnicki M, Accurso V, et al. Impairment of endothelium dependent vasodilation of resistance vessels in patients with obstructive sleep apnea. Circulation 2000; 102: 26072610.

28. Fujita T, Hemmi S, Kajiwara M, Yabuki M, Fuke Y, Satomura $\mathrm{A}$, et al. Complement-mediated chronic inflammation is associated with diabetic microvascular complication. Diabetes Metab Res Rev 2013; 29: 220-226.

29. Elmarakby AA, Sullivan JC, et al. Relation between oxidative stress and inflammatory cytokines in diabetic nephropathy. CardiovasTher 2012; 30: 49-59.

30. Vas PR, Ahluwalia R, Manas AB, Manu CA, Kavarthapu V, Edmonds $\mathrm{ME}$, et al. Undiagnosed severe sleep apnea and diabetic foot ulceration- a case series based hypothesis: a hitherto underemphasized factor in failure to heal. Diabet Med 2016; 33: 1-4.

31. Gabric K, Matetic A, Vilovic M, Ticinovic KT, Rusic D, Galic $\mathrm{T}$, et al. Health related quality of life in Type 2 diabetes mellitus patients with differential riskfor obstructive sleep apnea. Patient Prefer Adherence 2018; 12: 765-773.

32. Mok Y, Tan CW, Wong HS, How CH, Tan KL, Hsu PP, et al. Obstructive sleep apnea and type 2 diabetes: are they connected? Singapore Med J 2017; 58: 179-183.

33. Al Mawed S, Unruh M, et al. Diabetes, kidney disease and obstructive sleep apnea: a new frontier? CurrOpinPum Med 2016; 22:80-88.

34. Donovan LM, Ruschman M, Weng J, Basu N, Dudley KA, Bakker JP, et al. The efficacy of an obstructive sleep apnea screening and reatment program in patients with type 2 diabetes. Diabetes Res Clinprac 2017; 134: 145-152.

35. Ioja S, Chasens ER, $\mathrm{Ng}$ J, Strolo PG, Korytkowski MT, et al. Obstructive sleep apnea in adults with Type 1 and Type 2 diabetes : perspective from a quality improvement initiative in a university- based diabetic center. BMJ Open Diabetes Res Care 2017; 9: 433.

36. Zhu B, Ma C, Chaiard J, Sci C, et al. Effect of continuous positive airway pressure on glucose metabolism in adults with type 2 diabetes: a systematic review and metaanalysis of randomised control trials. Sleep Breath 2018; 22: 287-295.

37. Sharma SK, Agrawal S, Damodaran D, Sreenivas V, Kadhiravan T, Lakshmy $\mathrm{R}$. CPAP for the metabolic syndrome in patients with obstructive sleep apnea. N Engl J Med 2011; 365:2277-2286.

38. Malik JA, Mansoodi SR, Shoib S. obstructive sleep apnea in type 2 diabetes and impact of continuous positive airway pressure therapy on glycemic control. Indian JEndocrMetab 2017; 21: 106-112.

39. Martinez- Ceron E, Fernandez-Navarro I, Garcia- Rio F, et al. Efficacy of continuous positive airway pressure treatment on glucose metabolism in patients with obstructive sleep apnea. Sleep Med Rev 2016; 25: 121-130.

40. Chen L, Kuang J Pei JH, Chen HM, Li ZW, Yang HZ, et al. Continuous positive airway pressure and diabetes risk in sleep apnea patients: A systematic review and metaanalysis. Eur J Intern Med 2017; 39: 39-50.

41. Shechter A, Foster GD, Lang W, Reboussin DM, St-Onge MP, Zammit G, et al. Effects of a lifestyle intervention on REM sleep related OSA severity in obese individuals with Type 2 diabetes. J Sleep Res 2017; 26:47-755. 